\title{
L'ENQUÊTE SUR LES SAVOIRS AU TRAVAIL: ENTRE ACTIVITÉ NARRATIVE ET PROCESSUS DIALOGIQUES
}

\author{
Hervé Breton (Université de Tours, France)* \\ https://orcid.org/0000-0003-3536-566X \\ Daisy Moreira Cunha (UFMG)** \\ https://orcid.org/0000-0002-0702-8132
}

\section{RÉSUMÉ}

L’appréhension, la compréhension et la reconnaissance des savoirs incorporés et tacites qui circulent au sein des collectifs de travail supposent une forme d'enquête bien particulière au sein des approches ethnographiques : l'enquête narrative et dialogique, qui permet d'appréhender, à partir du travail réel, les savoirs au travail. Cette forme d'enquête sollicite l'expression du vécu, en première personne, dans le cadre des récits de pratique ou de la narration biographique, et en seconde personne lors des phases de mise en dialogue au sein des collectifs de recherche-formation. Elle permet d'appréhender le travail à partir de ses dimensions anthropologiques, impliquées et biographiques.

Mots clés: Dialogue. Enquête. Narration. Travail. Vécu.

\section{ABSTRACT}

\section{THE INQUIRY ON WORK KNOWLEDGE: BETWEEN NARRATIVE ACTIVITY AND DIALOGICAL PROCESSES}

The apprehension, understanding and recognition of the incorporated and tacit knowledge that circulates within work collectives presupposes a very particular form of investigation within ethnographic approaches: the narrative and dialogical investigation that makes it possible to apprehend, from real work, the knowledge at work. This form of investigation requires the expression of experience, in first person, as part of practice narratives or biographical narratives, and in second person, during the dialogue phases within research and training collectives. It allows us to understand the work from its anthropological, involved and biographical dimensions.

Keywords: Dialogue. Inquiry. Narrative. Work. Experience.

* Docteur en sciences de l'éducation et de la formation, université de Tours. Maître de conférences, EA7505, Université de Tours, France. E-mail: herve.breton.univ@gmail.com

** Doctorat en Philosophie, Université Aix-Marseille. Professeur Associé de la Faculté d'Éducation de l'Université Fédérale de Minas Gerais (UFMG), Brésil. Directrice de la Faculté d'Éducation de l'UFMG 2018-2022. E-mail: daisycunhaufmg@gmail.com 


\section{RESUMO}

\section{O INQUÉRITO SOBRE 0 CONHECIMENTO NO TRABALHO: ENTRE A ACTIVIDADE NARRATIVA E OS PROCESSOS DIALÓGICOS}

A apreensão, compreensão e reconhecimento do conhecimento incorporado e tácito que circula nos coletivos de trabalho pressupõem uma forma muito particular de investigação nas abordagens etnográficas: a investigação narrativa e dialógica que permite apreender, a partir do trabalho real, o conhecimento no trabalho. Esta forma de investigação requer a expressão da experiência, em primeira pessoa, como parte das narrativas da prática ou narrativas biográficas, e em segunda pessoa, durante as fases de diálogo dentro dos coletivos de pesquisa e formação. Permite-nos compreender a obra a partir das suas dimensões antropológicas, envolventes e biográficas.

Palavras-chave: Diálogo. Investigação. Narrativa. Trabalho. Experiência.

\section{Introduction}

L'appréhension, la compréhension et la reconnaissance des savoirs incorporés et tacites qui circulent au sein des collectifs de travail supposent une forme d'enquête bien particulière. Plusieurs obstacles viennent en effet rendre problématique le passage au langage de ce qui constitue les ressources de l'agir au travail, que ces ressources se traduisent concrètement dans des actions effectives ou qu'elles soient potentielles, telles les capacités qui s'actualisent (ou non) en fonction de la configuration singulière des situations. Dans les deux cas, en effet, les savoirs au travail se constatent d'abord en tant que résultat de et dans l'action, et non en tant que processus. Nous retrouvons ici le problème difficile des savoirs expérientiels, soit des savoirs à l'état pratique : ils se manifestent dans l'action en tant que résultats; ils sont transparents aux sujets, individuels ou collectifs, en tant que processus. C'est donc la pertinence des actions entreprises qui signalent (ou non) les savoirs détenus. Ces derniers peuvent donc être considérés comme «transparents» du fait de leur dimension tacite : qu'ils soient pensés à l'échelle individuelle ou collective, ils concourent silencieusement au déroulement de l'action sans pouvoir être saisis en tant qu'objets de pensée.
Le passage ci-dessous, extrait d'une recherche-action conduite à l'Université fédérale de Minas Gerais (UFMG) intitulée Connexao et Sabores, permet d'appréhender la complexité de la mise en mots et de la mise en dialogue entre les savoirs institués, souvent référencés, et les savoirs expérientiels, singuliers et contextuels, qui trouvent leur force de pertinence en contexte, tout en étant ontologiquement fragiles dès que les discours perdent de vue le travail réel et les situations concrètes:

Alors, nous parlons souvent comme ça, l'ingénieur... Comment êtes-vous parvenu à faire le bon choix... Oh, je l'ai fait... Vous ne lui expliquez pas que vous avez enlevé le carton découpé, que vous l'avez sorti, que vous avez mis un peu de colle, que vous l'avez fait passer par ici, que vous l'avez poussé par là. Vous n'expliquerez pas que vous lui avez fait tout ça... Vous dites : je l'ai fait et c'est tout, parce qu'il vous a dit de le faire. Il donnera l'ordre : faites-le ici. Mais il ne vous demandera pas, écoutez, si la soudure est de deux millimètres de plus, vous le faites comme ça, si elle est de deux millimètres de moins, elle est grillée. Si le tube faisait $15 \mathrm{~cm}$ de long, au moment où vous l'avez soudé, combien ya-t-il de soudures, l'une appuyée contre l'autre, la plaque, elle rétrécit, non? Quand elle rétrécit, cela change toutes les mesures que vous avez faites. Seulement dans son dessin, cela n'apparaît pas toujours. Il y a un ingénieur qui ne le sait 
même pas. Il ne le prend pas en compte, alors, lorsque la plaque diminue, il n'y a aucun moyen de la ramener chez elle, c'est tout, c'est fini. Alors ça, c'est le truc avec l'ingénieur. D'après ce que je sais... en conflit avec l'ingénieur. Et c'est un vrai différend, de connaissance. (CUNHA, 2007, p. 111). ${ }^{1}$

Le passage ci-dessus manifeste une dialectique entre deux formes de raisonnement, l'une théorique, l'autre expérientielle. L'appréhension de cette dialectique est rendue possible par l'expression d'un récit, celui d'un mineur de la CNTSM qui, dans le cadre d'une recherche-action sur le travail, mets en mots une situation concrète régie par l'écart des points de vue sur la manière de concevoir l'activité. Ce sont ces dynamiques de composition et de mise en dialogue sur les savoirs au travail que nous proposons d'examiner dans cet article. La proposition est ici la suivante: l'appréhension des savoirs expérientiels suppose d'accompagner une dynamique qui procède d'un passage, celui de l'expérience au langage. Ce passage durant lequel l'expérience devient dicible, trouve les mots pour se dire (BOUTET, 2005), s'accompagne. Paradoxalement il faut, pour accompagner l'expression du vécu au travail, que cette expression soit en première ou deuxième personne en mobilisant des procédés qui permettent de dépasser les discours génériques et globaux sur le travail.

C'est l'objet de cet article que d'examiner les formes d'enquête sur les savoirs au travail qui permettent d'accéder au « réel du travail » lors de la mise en mots. Deux formes d'enquête sont ici étudiées, leur dimension commune étant d'accompagner et d'organiser la mise en mots du vécu au travail afin, notamment, de permettre l'appréhension des savoirs qui circulent «à l'état pratique ». Elles supposent toutes deux un travail durant lequel les professionnels « entrent en enquête » sur leurs savoirs, via la narration du vécu (BRETON,

1 Mineur de la Confederação Nacional dos Trabalhadores do Setor Mineral (CNTSM), qui rassemblait, à l'époque, les syndicats de travailleurs du secteur minier au Brésil.
2018) et via la mise en dialogue de l'expérience vécue au travail au sein de collectifs dans le cadre d'une recherche-action.

Les verbatims présentés dans cet article ont été collectés dans le cadre d'un dispositif de recherche-formation-action avec des salariés mineurs au Brésil qui comprend trois périodes:

- 2005-2008: « Connexions des connaissances sur le travail avec les travailleurs des mines » (financement du ministère du Travail, Brésil);

- 2008-2010: «Connexions de travail dans le secteur de l'énergie avec les travailleurs du Companhia Energética de Minas Gerais (CEMIG) dans le secteur électrique » (financement Sindieletro-MG);

- 2011-2014: "Connexions en matière de santé et de sécurité dans le secteur minier» (durant laquelle le public a été élargi pour intégrer les salariés des mines, des ports et de l'électricité).

Ces dispositifs ont été mis en place avec beaucoup de difficultés du fait de la distance à la fois géographique et socio-professionnelle entre les chercheurs de l'université et les conditions d'exercice des participants qui, pour la plupart, ne bénéficiaient d'aucune aide ou allégement du temps de travail pour participer à la recherche. Ainsi, en l'absence de droit à la formation continue au Brésil, les rencontres ont dû avoir lieu durant le weekend. Par ailleurs, durant ces rencontres, il a fallu surmonter les défis épistémologiques soulevés dans le collectif en organisant des espaces de confrontations et de discussions pour le croisement des savoirs, de manière multi, inter, pluri, transdisciplinaire $\mathrm{du}$ fait des questions soulevées au sein du collectif, composé des salariés et des chercheurs. La dynamique de ces rencontres favorise un espace ouvert, en partie libéré des contraintes traditionnelles rencontrées dans les situations de travail des salariés. Selon une perspective méthodologique qui dépasse le cadre de recherche traditionnelle de nature qualitative, la parole libre doit être 
assurée dans un espace de circulation des connaissances et des valeurs qui composent l'expérience des participants. La création d'un espace d'expression propice aux narrations et l'établissement d'un dialogue sur le travail à partir d'une pluralité de situations ont pour objet de favoriser l'expression du vécu à partir du réel du travail. Dans ce cadre, tous les point de vue doivent ainsi pouvoir être exprimés, écoutés et interrogés à partir des questions émergentes sur le vécu au travail dans les mines du fait des espaces de parole ainsi ouverts. La narration du vécu et sa mise en dialogue peuvent alors prendre plusieurs directions et poursuivre plusieurs objets : par les illustrations et témoignages présents dans les récits, les connaissances et valeurs peuvent circuler; par l'analyse des incidents et accidents, les conditions réelles d'exercice peuvent être analysées et les relations sociales qui préfigurent la vie au travail interrogées, en vue notamment d'actions collectives concertées et communes.

\section{Enquête ethnographique et enquête narrative}

Si l'enquête ethnographique procède selon Laplantine (1996, p. 15) d'un « dépaysement » - « Cette expérience, à vrai dire étrange, qui consiste à nous étonner de ce qui est le plus familier [...] et à rendre plus familier ce qui nous paraissait originellement étrange et étranger [...] est l'expérience même de l'ethnographie ou, comme l'on dit encore, du terrain »-, elle suppose pour être exercée de voir et de regarder: « La perception ethnographique n'est pas, quant à elle, de l'ordre de l'immédiateté de la vue, de la connaissance fulgurante de l'intuition, mais de la vision (et par conséquent de la connaissance) médiatisée, distanciée, différée, réévaluée, instrumentée (stylo, magnétophone, appareil photographique, caméra...) et dans tous les cas retravaillés par l'écriture » (LAPLANTINE, 1996, p. 17). Ainsi, à la différence de l'enquête ethnographique qui suppose d'exercer son regard sur les situations, « un regard si ce n'est inquiet, du moins questionnant, qui part à la recherche de la signification des variations » (LAPLANTINE, 1996, p. 17), l'enquête narrative suppose d'exercer au travers de la texture de l'expérience qui se donne au souvenir, les dimensions, fragments et aspects du vécu à partir desquels le discours peut s'élaborer, une manière d'appréhender les tensions dialectiques relatives aux dynamiques par lesquelles les «points de vue » s'édifient (FRUTEAU DE LACLOS, 2016), les manières de dire et de faire s'inventent (DE CERTEAU, 1990), les formes de récits au travail se composent (BRUNER, 1986).

Cette perception de la texture de la dimension prélangagière et langagière du vécu entretient cependant des dimensions communes avec la perspective ethnographique, à partir de ce que Merleau-Ponty a nommé « le visible et l'invisible » de l'expérience : « Il faut nous habituer que tout visible est taillé dans le tangible, tout être tactile promis en quelque manière à la visibilité, et qu'il y a empiètement, enjambement, non seulement entre le touché et le touchant, mais aussi entre le tangible et le visible qui est incrusté en lui, comme inversement, lui-même n'est pas un néant de visibilité, n'est pas sans existence visuelle » (MERLEAUPONTY, 1993, p. 177). Cette citation proposée par Laplantine, qu'il applique à l'enquête ethnographique, trouve la même pertinence pour penser la singularité de l'enquête narrative et dialogique. La narration du vécu peut être définie comme une activité initiée par des sujets individuels ou collectifs qui saisissent des moments vécus au travail, des périodes de vie inscrites dans leurs parcours professionnels, des expériences collectives d'épreuves partagées dans le cadre du travail, pour les mettre en mots et les exprimer. Et c'est du fait de l'exercice narratif que les dimensions non visibles du travail se découvrent. De ce point de vue, la narration du vécu au travail, en procédant au passage de l'expérience au langage, participe de l'émergence et de la constitution des objets 
de pensée, de la formalisation des savoirs, d'une connaissance qui s'origine et trouve ses ancrages dans la sphère « expérientielle ». Le dépaysement évoqué par Laplantine peut donc être traduit en termes de " renversement ». Ainsi, si comme l'examine Vermersch à partir des actes de réduction par lesquels sont saisis l'expérience, la structure habituelle à partir de laquelle le discours s'établit de manière séquencée sur le mode "vécu-langage-pensée », cette séquence est inversée au cours de l'enquête narrative, qui suppose, selon Vermersch, « une réduction de la mise en langage: vécu-langage-pensée dans laquelle il y a accueil du vécu avant toute chose » (VERMERSCH, 2003, p. 243). Cette dimension qui procède de l'accueil du vécu constitue le sol commun de de l'enquête narrative, qu'elle soit conduite en première personne, par la description et/ ou la narration biographique du vécu, ou en seconde personne, par le dialogue, au cours d'échanges et de conversations au sein des collectifs de travail.

\section{Enquête sur les savoirs au}

\section{travail à partir de la narration biographique}

Chico n'est pas ingénieur en mécanique, mais il est ingénieur. Si je nous attrape, moi et Chico, je suis chaudronnier et lui ingénieur, à parler comme ça, oh... Nous devons passer un tuyau. Il doit rester contre le mur tout le temps et il doit passer d'ici à là sans enlever cette poutre de sa place. C'est une autre façon de travailler. Il le voudra, il calculera l'angle, il calculera l'angle du rayon, il calculera combien de millimètres sont d'ici à là, il étendra la plaque, etc. Si je suis dans un endroit et que le gars parle comme ça, je ne perdrai pas de temps, je dois produire, non? J'y ai mis un morceau de papier, je l'ai découpé, je l'ai mis sur le dessus de l'assiette et je l'ai découpé. Je ne vais pas continuer à calculer, parce que je n'ai pas le temps de faire ça. Si je continue à calculer... Le responsable... Mettez mon... Vous avez regardé ça pendant quatre heures, vous l'avez fait et ce n'est pas encore fini ? Parce que la fonction du travailleur n'est pas de dessiner.
Faire les dessins, c'est pour l'ingénieur. Parce que nous le faisons plusieurs fois... Il a fait tous les calculs, milliseconde par milliseconde, mais la plaque quand vous la pliez, elle change. Quand le soudeur soude ou qu'il faut se souder soi-même, quand on soude le millimètre change là, un millimètre va là ou là. Au moment où vous rentrez là-dedans, ça ne marchera pas si vous suivez ses mesures. Normalement. Pourquoi ? Parce que ces millimètres changent la soudure, elle peut devenir un peu plus grande ou un peu plus petite et il n'y a aucun moyen de mettre la ponceuse ou de réduire le millimètre dans la soudure, on en perd la puissance. Si la plaque dépasse de deux millimètres la taille de la ponceuse et que je la coupe, vous ne pouvez pas le faire, vous avez coupé l'alimentation de la soudure, vous avez tué tout le travail. Tu l'affaiblis. Donc tu dois la mettre là-dedans. Et qu'est-ce que tu vas utiliser? Vous prenez une plaque plus mince, si la plaque est trop épaisse, vous prenez un carton, vous y allez, vous coupez la courbe et vous la posez sur le dessus (CUNHA, 2007, p. 111).

La problématique rencontrée lorsqu'un sujet, un collectif de travail, un professionnel cherche à mettre en mots ses savoirs est qu'il n'en dispose pas en tant qu'objets de pensée. Ce point apparaît de manière concrète dans l'extrait de récit du mineur brésilien présenté ci-dessus: la mise en mots porte sur l'activité conduite, les raisonnements tenus... Ce qui transparaît du récit, au-delà des actes et des gestes réalisés pour poser un tuyau, c'est une capacité à lire la situation, à " percevoir » et à " sentir », en fonction des contextes matériels et physiques concrets dans lesquels cette activité doit être réalisée, les manières de faire les plus pertinentes. Du point de vue du mineiros la pertinence est conçue en étroite adéquation avec le «donné » de la situation. Les procédés mobilisent l'adhésion maximale avec la situation, à partir de gestes qui s'ajustent au plus près de ce que les circonstances rendent nécessaire: " J'y ai mis un morceau de papier, je l'ai découpé, je l'ai mis sur le dessus de l'assiette et je l'ai découpé. Je ne vais pas continuer à calculer, parce que je n'ai pas le temps de faire ça. 
Si je continue à calculer » (CUNHA, 2007, p. 111). Les savoirs de l'expérience ont en effet cette caractéristique : ils trouvent leur pertinence en contexte, par l'invention de procédés régis par la faisabilité, l'économie des ressources et le souci de soi. Ce régime d'activité (BILLETER, 2002) suppose une attention aux données du contexte et mobilise des inférences abductives et transductives (DENOYEL, 1999) pour inventer et agir en fonction du nécessaire. C'est ce qui explique la dialectique entre les formes de savoirs qui traversent l'ensemble du récit: face aux savoir-faire procéduraux de l'ingénieur, le mineur revendique la valeur des savoirs d'expérience, dont la force et la pertinence sont de pouvoir prendre en compte ce qui se donne dans la situation de manière immédiate - « Nous devons passer un tuyau et il doit rester contre le mur tout le temps et il doit passer d'ici à là sans enlever cette poutre de sa place » (CUNHA, 2007, p. 111) -, mais également dans une dynamique d'anticipation : "Parce que ces millimètres changent la soudure, elle peut devenir un peu plus grande ou un peu plus petite et il n'y a aucun moyen de mettre la ponceuse ou de réduire le millimètre dans la soudure, on en perd la puissance. » (CUNHA, 2007, p. 111). Cette forme d'expression du vécu au travail permet d'appréhender le vital qui circule au cours et au gré des situations de travail : "Parce que ces millimètres changent la soudure, elle peut devenir un peu plus grande ou un peu plus petite et il n'y a aucun moyen de mettre la ponceuse ou de réduire le millimètre dans la soudure, on en perd la puissance. Si elle dépasse de deux millimètres la taille de la ponceuse et que je la coupe, vous ne pouvez pas le faire, vous avez coupé l'alimentation de la soudure, vous avez tué tout le travail. Tu l'affaiblis. Donc tu dois la mettre là-dedans et qu'est-ce que tu vas utiliser ? Vous prenez une plaque plus mince, si la plaque est trop épaisse, vous prenez un carton, vous y allez, vous coupez la courbe et vous la posez sur le dessus. » (CUNHA, 2007, p.111).

\section{Le « problème facile » des}

\section{savoirs au travail : réduire le vécu au travail aux dimensions opératoires de l'activité}

La réduction de la complexité de la vie au travail aux seuls domaines opératoires et procéduraux de l'action relève de ce que Chalmers (1995) a nommé le «fossé explicatif » : elle cherche à comprendre les pratiques en prenant comme «donnée exclusive » l'intentionnalité du sujet. La centration sur l'agir et ses fondements logiques conduit à penser les processus de couplage entre la perception du sujet et le réel de la situation donnée à partir d'un mouvement par lequel le réel de la pratique est appréhendé en tant qu'objet de pensée. Selon cette perspective, le problème posé par les « savoirs expérientiels » est contenu dans le couplage « action-conceptualisation »de toute pratique professionnelle. Pour Pastré (1999), les invariants opératoires sont des " conceptualisations à l'état pratique ». L'enjeu est alors de chercher à comprendre et à accompagner les processus qui permettent la saisie de la pratique par la pensée, et ainsi de l'action au concept. Une certaine orientation téléologique imprègne donc ce type de démarche : elle consiste en effet à proposer l'idée que la conceptualisation est un accomplissement nécessaire de l'action et que, par voie de conséquence, l'expérience non conceptualisée demeure une matière brute, informe, telle une substance qui reste en attente de raffinage. Cette approche substantialiste de l'expérience qui conduit à la traiter comme une matière dont il serait possible d'évaluer la qualité et les limites caractérise le " problème facile » de l'expérience. Cette logique fait porter l'attention au cours de l'enquête sur les savoirs expérientiels sur les dimensions intentionnelles et finalisées de l'agir professionnel. Il est cependant possible de considérer que les visées intentionnelles qui orientent l'action en situation ne résument pas l'agir au travail, 
voire qu'il constitue une donnée qui permet de comprendre les dimensions relevant de l'inscription et de l'implication des sujets au travail. Ainsi, le problème facile de l'expérience consiste à réduire le travail à ce qui relève de la dimension finalisée de l'action. Elle investigue en effet les contenus de l'expérience et le rapport du sujet à ces contenus, que celle-ci soit conduite à partir des savoirs tacites et incorporés qui se donnent au sujet sur le mode tacite en fonction de la situation éprouvée et/ou de schèmes conscientisés relatifs à la mise en œuvre d'une pratique. Cette approche comporte cependant ses limites car elle laisse quasiment dans l'ombre les dimensions autopoïétiques (VARELA, 2017) de toute expérience vécue. C'est cet aspect qui caractérise le « problème difficile » de l'expérience.

\section{Le « problème difficile » du vécu au travail : appréhender selon une perspective expérientielle et biographique les processus de constitution et d'évolution des savoirs d'expérience}

Comment décrire l'activité dans les situations de travail et ainsi reconnaître les savoirs expérientiels d'un sujet, d'un professionnel, d'un collectif de travail, sans les réduire à leur seule dimension opératoire et finalisée ? Il est en effet raisonnable de soutenir l'idée que ce qui constitue une ressource de l'agir (les savoirs de l'expérience) s'est constitué dans la durée de la vie du sujet (dimension historique et biographique) et participe en même temps, selon des modes à préciser, des anticipations quant au devenir (dimension prospective). De ce point de vue, les savoirs expérientiels sont bien plus que des moyens au service d'une fin. Il apparaît en effet extrêmement réducteur de considérer que la capacité à jouer du violon ne sert qu'à jouer des morceaux de musique. En effet, savoir jouer d'un instrument de musique permet de vivre des expériences esthétiques, d'en faire vivre d'autres à autrui et, au-delà, d'habiter le monde en intégrant l'esthétique des sons, le rythme des mélodies, la synchronicité des collectifs de musiciens. Autre exemple, peut-être moins évident au premier abord : savoir servir en salle, dans un restaurant, une brasserie ou un café, constitue une ressource bien plus vaste que celle d'acheminer des mets ou des boissons sur des tables. Au-delà des gestes et des actes, une conception du service s'affirme, qui comporte des dimensions d'accueil au sein d'un lieu propice à la détente, à la réflexion, aux échanges entre pairs, durant les différents temps de la journée. Il est donc possible de déplorer le caractère réducteur d'une approche des savoirs expérientiels qui réduit l'agir à ses dimensions instrumentales dont la caractéristique est de n'être régie que par le rapport moyens/fins. Ce constat étant posé, le problème reste cependant entier. Il est en effet difficile d'appréhender par la pensée, et donc de mettre en mots, ce qui circule et traverse chacun des gestes professionnels, ce qui dépasse chaque acte et qui relève des valeurs et de l'histoire du sujet qui les réalise. Cette perspective suppose en effet de porter attention à la vie intentionnelle du sujet, mais également aux modes de donation des situations (VARELA; THOMPSON; ROSCH, 2003) et aux conceptions du sujet sur la manière juste et pertinente d'exercer son métier. De ce point de vue, les savoirs expérientiels sont pensés en tant que processus résultant d'une réciprocité dynamique entre « situation perçue » et « agentivité du sujet ». Cette conception des savoirs expérientiels n'est pas sans conséquence sur les logiques d'enquête qui permettent de les appréhender. La problématique est alors la suivante : les savoirs n'étant pas détenus en propre mais seulement disponibles au gré des situations, leur caractérisation suppose la narration de l'agir à partir de moments vécus, afin que puissent être dits et restitués les manières de faire, l'ajustement des manières de faire en 
fonction des horizons de perception du sujet en situation, et les formes d'implication du sujet dans ses pratiques, son parcours professionnel et son métier. La prise en compte de ces différents paramètres suppose un travail de mise en mots et en sens des pratiques professionnelles qui puisse conjuguer les régimes de la description phénoménologique et de la narration biographique (BRETON, [2020?]).

\section{Comprendre le travail via l'enquête dialogique}

Je vais vous parler de l'expérience à Santa Suzana, du feldspath et de l'aluminium. La question de l'humidité est venue du gars qui était là, à l'excavatrice, lorsqu'il a jeté le matériau dans le concasseur primaire. Cet ouvrier savait quand le matériau était mouillé ou sec et c'est lui qui a extrait le produit à son arrivée sur le chantier. Là, c'était un centre de meunerie, les produits arrivaient du Minas Gerais, de São Paulo ou même de Bahia. Juste en regardant, il sentait si le matériau était bon ou s'il fallait le mélanger avec le matériau sec ou humide. À l'époque où il n'était pas rentable d'acheter le matériel, ici dans le Minas Gerais, les gens ont décidé d'acheter le feldspath dans une région de São Paulo. C'était un endroit où plusieurs carrières ont été coupées et il y avait du feldspath au milieu des concasseurs. Qui prendraient-ils pour savoir si le matériel est de bonne qualité ou non ? S'ils ont pris le géologue, il n'avait aucune expérience. Alors, qui prendront-ils ? L'opérateur de la pelle. Il a dit que le matériel était de mauvaise qualité, mais si nous prenions 40 p. 100 de ce matériel plus 60 p. 100 de notre stock, cela ferait un produit de bonne qualité. C'était juste un opérateur. Il a pris sa retraite et a continué à travailler parce que son expertise était trop grande. (CUNHA, 2007, p. 109).

L'extrait ci-dessus présente le témoignage d'un mineur qui fait état d'un savoir expérientiel d'un de ses collègues qui pouvait, par les sens, identifier et évaluer la qualité d'un minerai. Les connaissances du feldspath de ce mineur du Minas Gerais étaient tellement profondes qu'il pouvait « à l'œil » connaître ses propriétés les plus importantes (ces pro- cédés nécessitant d’ordinaire des analyses de laboratoire). Cette capacité rendue possible par l'acquisition de « savoirs à l'état pratique » contient, si elle doit être décrite de manière formelle, une somme de connaissances considérables. En effet, le feldspath est une des grandes familles de minéraux dont les caractéristiques physiques et chimiques sont communes alors qu'elles peuvent comporter des variations de couleur. Du point de vue chimique, ces « aluminosilicates » peuvent être potassiques (K), calciques (Ca) ou sodiques ( $\mathrm{Na}$ )... Ce minéral est très utilisé dans l'usinage de céramiques de verre, ainsi que dans l'industrie de la teinture. La composition chimique du minéral est une question stratégique pour les industries qui l'utilisent. Cette composition chimique est reflétée par sa couleur, les cristaux et leurs facettes, ses arêtes suite aux brisures; sa brillance et son poids constituent des repères utilisés par les ouvriers pour identifier si le feldspath contient de la potasse calcique ou du sodium. Ces éléments théoriques relevant du savoir de la science géologique étaient appréhendés et connus par l'ouvrier mineur sur le plan pratique et expérientiel, sans qu'il puisse en faire état sur une plan analytique et formel. Ce n'est qu'au cours de l'échange, lors de la discussion et du témoignage recueilli par la recherche-action, que la capacité est suggérée et que les savoirs sont indiqués, de manière allusive.

Le court récit a été recueilli dans le cadre de la formation-recherche Conexões de saberes sobre trabalho no setor mineral (ADAO et al., 2008). Dans le dialogue interlocutif entre pairs, les échanges s'amorcent par de micro-récits (GEORGAKOPOULOU, 2007; MONTANDON, 2013) qui condensent de manière allusive les savoirs expérientiels détenus par les mineurs. La circulation des micro-récits s'opère ensuite par le dialogue, qui sollicite la mémoire d'un autre mineur qui, à son tour, témoigne de son vécu, celui-ci venant, lorsqu'il prend la parole, nourrir les échanges. Au fil des discussions entre travailleurs, ainsi qu'avec les chercheurs, 
la socialisation des vécus singuliers au travail intensifie la mise en commun des savoirs, ce qui permet d'intensifier la compréhension mutuelle. Ainsi, par le dialogue interlocutif, d'autres histoires sont partagées dans la conversation sur le même sujet, ce qui permet de préciser les connaissances, d'exprimer les valeurs et les stratégies d'action nécessaires pour résoudre les problèmes rencontrés du fait de l'organisation parcellaire du travail. Cette forme de dialogue conjugue différentes dynamiques : entre pairs, la compréhension se fait parfois par des « mots du métier » qu'ils sont les seuls à comprendre. Elle permet au cours de l'échange de préciser « chemin faisant » les termes, de diversifier les énoncés et les manières de dire, d'initier une activité réflexive et dialogique afin de chercher collectivement des manières de dire qui fassent sens à l'échelle du collectif. Ce travail de compréhension réciproque peut demander un étayage de connaissances du fait de "l'inconfort intellectuel» (SCHWARTZ, 2000b) résultant de la prise de conscience des formes des savoirs circulant au sein du collectif. Ainsi, les chercheurs ont dû collecter des renseignements sur les minéraux afin de pouvoir comprendre les enjeux des savoirs portés par les mineurs qui savaient reconnaître le feldspath.

\section{Sources et ancrages de l'enquête dialogique au travail}

La perspective théorico-méthodologique s'inscrit bien dans l'héritage de Paulo Freire (1996a) et de l'éducation populaire dans la mesure où il y a un appel au dialogue permanent avec l'expérience de vie et le travail préalable des élèves adultes, puisque porteurs de savoirs et valeurs. Chez Freire, le dialogue permet l'écoute attentive, par l'échange de savoirs, des modes de vivre et de sentir les circonstances historiques dans lesquelles les sujets sont plongés. Dans ces échanges dialogiques d'expériences de vie, et de travail, il est possible d'appréhender l'humanité indus- trieuse. Le dialogue sur l'expérience est aussi au centre des approches contemporaines du travail. Chez Oddone, en Italie, vers 1970, les « communautés scientifiques élargies » partent de l'expérience de travail pour réinterroger les connaissances et les normes de vie en commun dans les organisations productives, mais aussi dans les formes de gouvernement de la cité. L'expérience est comprise à partir de ce qu'elles faisaient, pensaient du sens attribué au travail, ainsi que des objectifs de leurs luttes pour la recherche de meilleures conditions de vie et de travail. L'expérience a été comprise comme une manière de faire l'expérience, aux niveaux individuel et collectif, des difficultés que posent les situations de travail, dans l'accomplissement des tâches, dans la capacité à faire face aux problèmes et à trouver des solutions aux problèmes concrets rencontrés au quotidien dans les tâches prescrites, tout en trouvant des moyens de résister au gouvernement tayloriste par l'expérience, y compris sur le plan politique et syndical (ODDONE; BRIANTE, 1981, p. 17). Pour Oddone (ODDONE; BRIANTE, 1981, P. 59-60), l'expérience se forge dans la confrontation des conditions générales de production dans les situations de travail dans lesquelles le travailleur vit: « En établissant cette jonction au sein de l'entreprise entre organisation formelle et organisation réelle, le travailleur acquiert une expérience en matière de tâche, mais en transmettant son savoir à d'autres il en acquiert également une se rapportant aux hommes. Pourtant, on peut affirmer que la formation informelle est le fruit de deux expériences : d'une part, de l'expérience purement et simplement transmise et, d'autre part, de l'expérience née des difficultés rencontrées et surmontées au cours de la transmission. Il émerge de ce deuxième type d'expérience un grand nombre de nouveaux problèmes encore sans solution et qui, de plus, ne sont pas même perçus puisque leur objet diffère de celui des problèmes initiaux (machine ou tâche) ».

Différentes méthodes peuvent être mobilisées pour favoriser cette mise en dialogue 
des savoirs au travail. L'« instruction au sosie» consiste à demander de communiquer son expérience à quelqu'un qui va nous remplacer, d'où ressort l'expérience du vécu, singulier et collectif, tel que l'avait développé Oddone à la FIAT à Turin dans les années 1970. Ces narrations du vécu sont des matériaux que les chercheurs doivent prendre comme source de réflexion pour repenser les modèles théoriques sous-jacents à leurs recherches. Ainsi, les travaux pionniers d'Oddone et Briant (1981) soulignent les possibilités d'articulation, dans une confrontation permanente, des connaissances scientifiques et de l'expérience des travailleurs.

De même, Alain Wisner et son équipe, à la même époque, essaient de comprendre la nécessité d'appréhender le travail en situations concrètes pour le transformer à travers une confrontation permanente entre le travail prescrit et le travail réel, ce qui ramène à une confrontation épistémologique permanente entre le planifié et l'exécuté. Ainsi, l'enquête narrative orientée vers le dialogue peut également être pensée à partir de cette dialectique entre les dimensions prescrites du travail et les pratiques réelles déployées en contexte : cette enquête est alors l'occasion de l'expression et de la circulation des points de vue des sujets faisant l'expérience du travail dans les collectifs et entreprises. Dans ce sens, le livre Comprendre pour transformer (GUÉRIN et al., 2007) est un traité qui expose progressivement ces règles, ces principes, ces façons de procéder dans le cas de l'analyse ergonomique du travail en vue de construire des interventions en situation de travail. L'un des enjeux poursuivis par cette forme d'enquête est de limiter les phénomènes de réduction résultant d'une appréhension du réel à partir des « découpages disciplinaires ». Ainsi, par la tension dialectique entre problèmes de l'expérience et problèmes de recherche, de nombreux aspects des phénomènes vécus au travail peuvent rester inaperçus du fait du maintien par les chercheurs de grilles de lecture adossées aux catégories des savoirs disciplinaires et/ ou académiques. La puissance résultant de la conjonction du narratif et du dialogique est de permettre la circulation des manières de dire et ainsi d'instaurer des rapports de proximité entre les savoirs théoriques du travail prescrit et les savoirs d'action du travail réel. La mise au jour de ces dialectiques est l'un des enjeux de l'enquête dialogique au travail.

\section{L'enquête dialogique et ses processus de reconnaissance des savoirs au travail}

Avec Bakhtine, nous avons également appris le plurilinguisme dialogique comme moyen de préserver la diversité des voix discursives. Une caractéristique fondamentale de toute énonciation est qu'elle est soutenue par des positions sociales. Ces discours circulent socialement, avec des points de vue axiologiquement marqués sur les choses de ce monde, représentant des synthèses entre différentes langues de toutes sortes de groupements socioculturels et économiques, de professionnels, générationnels, de genre... Ces discours sont matérialisés dans différents dialectes, différentes langues, exprimant des sentiments du monde et des tendances historiques en événements concrets lors de situations professionnelles. La diversité qui marque ce plurilinguisme est avant tout sociale, culturelle, et donc historique et économique, située là où se trouve celui qui parle, représentant les forces de la lutte locale où il vit et travaille. Le sauvetage de cette énonciation, sous forme dialogique, dans des dispositifs comme le nôtre fait émerger la pluralité des voix présentes dans les situations de travail dans le monde minier. À travers des histoires et des dialogues nous pouvons entrevoir les luttes en cours ainsi que, d'une certaine manière, comment cette expérience est vécue par ceux qui y participent. Nous pouvons entrevoir les connaissances et les valeurs qui opèrent dans les situations. 
Mais le dialogue sur le travail entre chercheurs et mineurs dans notre dispositif Conexões de saberes sobre trabalho no setor mineral (ADAO et al., 2008) n'était pas évident car, dans cette expérience individuelle et collective, leurs valeurs, leurs connaissances, et leur capacité à intervenir dans la résolution des problèmes qui se posent au travail ne sont pas facilement communicables. Ainsi, dans l'extrait proposé, différentes formes de dialogues peuvent être identifiées : le mineur témoigne de savoirs détenus par un de ses collègues. Il procède pour cela d'une saisie de vécu situé dans son histoire (dialogue intralocutif) pour dire cette expérience et les savoirs du collègue au groupe de pairs (collectif de travail) qui connaissent de manière expérientielle les savoirs dont il est question. Il socialise également (et dans le même temps) ces savoirs aux chercheurs et experts, qui eux doivent apprendre théoriquement les savoirs géologiques dont il est question pour les comprendre et ainsi rendre possible le maintien du dialogue.

\section{Principes et méthode de l'enquête narrative et croisement entre le biographique et le dialogique}

\section{De l'expérience aux questions de méthode}

Il n'y a pas de moyen sûr. Selon l'origine latine du terme methodus, le préfixe meta signifie « à travers » et hodos « chemin », « route », ce qui signifie donc, étymologiquement, « un chemin par lequel nous cherchons à atteindre ou à réaliser quelque chose ». Il est également possible de considérer le chemin comme un ensemble de procédures qui suit l'esprit pour découvrir et démontrer la vérité (dans les sciences). Ou, dans un sens proposé par la logique, comme une manière de conduire la raison dans la recherche de la vérité en science. Il peut s'agir d'une méthode d'analyse, il est donc logique d'être guidé par le découpage de cette expérience en parties/dimensions. Cela nécessiterait alors l'utilisation de la méthode synthétique pour recomposer le tout par les composants configurés analytiquement. Mais le problème est que, dans le cas de l'expérience de travail, le tout ne peut jamais être la somme des parties symétriquement découpées et analysées, pour au moins deux raisons: les parties ont été prises comme des configurations séparées de manière générique et les "sciences » qui analysent avec leurs instruments ne traitent pas ces parties par des protocoles méthodologiques qui les combinent en unités. Ainsi, il est nécessaire de considérer avec attention les apports résultant de la narration du vécu et de la mise en dialogue de l'expérience au travail.

\section{La force de l'enquête narrative : mise en mots et mise en intrigue des savoirs}

La caractéristique de la narration est de pouvoir appréhender l'expérience en limitant les processus de réduction des dimensions organique et biographique qui composent l'activité: organique car, nous avons essayé de le montrer, l'agir et ses ressources (soit les savoirs expérientiels ou savoirs pratiques) ne peuvent être appréhendés en dehors (d'un point de vue d'extériorité) de la situation dans laquelle ils s'exercent ; biographique, car l'activité en situation de travail se déploie en fonction d'un style, celui du sujet au travail et du collectif dans lequel il évolue. C'est la force du récit que de pouvoir appréhender et tenir ensemble ces dimensions contradictoires. Il faut, pour comprendre ces aspects, revenir à ce que Ricoeur nomme le pouvoir de configuration du récit: « L'acte de mise en intrigue combine dans des proportions variables deux dimensions temporelles : l'une chronologique, l'autre non chronologique. La première constitue la dimension épisodique du récit : elle caractérise l'histoire en tant que faite d'événements. La seconde est la dimension configurante proprement dite, grâce à laquelle l'intrigue transforme les événements en histoire » (RICOEUR, 1983, p. 129). Cette capacité de la narration d'alterner entre 
le régime de la description détaillée qui permet la mise en mots des formes d'agir en situation et celui de la narration de la durée qui permet la mise en mots des transformations qualitatives des manières de faire (dynamique de professionnalisation) et des manières de voir (édification des valeurs) dans le temps confère à l'activité narrative une puissance.

C'est à partir de ce pouvoir configurant du récit et des possibilités qu'il offre en termes de manifestation des savoirs d'expérience, de mise au jour des connaissances du point de vue du sujet sur le travail et de formation de soi que les courants des histoires de vie en formation (PINEAU; LEGRAND, 2019) et de la recherche biographique (DELORY-MOMBERGER, 2010) se sont déployés en Europe (GONZÁLEZ MONTEAGUDO, 2008) et au Brésil (SOUZA, 2008), notamment. La forme narrative de l'enquête, qu'elle soit individuelle ou collective, conduite avec soi-même par " conversion du regard » ou dans le cadre d'interactions au sein d'un collectif, comporte une dimension dialogique: « Toute référence est co-référence dialogique ou dialogale. Il n'y a pas à choisir entre une esthétique de la réception et une ontologie de l'œuvre d'art. Ce que reçoit le lecteur, c'est non seulement le sens de l'œuvre mais aussi, à travers son sens, sa référence, c'est-à-dire l'expérience qu'elle porte au langage et, à titre ultime, le monde et sa temporalité qu'elle déploie en face d'elle » (RICOEUR, 1983, p. 147).

\section{La force de l'enquête dialogique : de la réciprocité à la mutualité dans la narration du vécu au travail}

La connaissance qui circule au travail est d'abord et avant tout personnelle, produite dans l'histoire de vie de chaque personne, par rapport aux autres et aux situations de vie. Selon Bernard Charlot ce rapport est à la fois épistémique, identitaire et social. Il est épistémique parce que c'est une façon de comprendre le monde, qui peut être théorique mais qui révèle aussi des manipulations d'objets, le développement d'une activité, des comportements et des relations avec les autres, en particulier. Les processus d'apprentissage sont, selon cette perspective, le résultat des histoires du sujet, de sa vision du monde, de ses principes et de ses valeurs, en d'autres termes, de formes de reconnaissance réciproques qui fondent les collectifs et les rapports coopératifs au travail. Par conséquent, toute relation avec la connaissance est aussi une relation avec l'autre : l'autre qui favorise l'apprentissage, qui contemple ou s'oppose, ou même l'autre idéalisé (CHARLOT, 1999). Enfin, toute relation avec la connaissance est une relation sociale, car le sujet qui apprend fait partie d'un monde rempli de relations sociales. Ce sujet occupe une place sociale, porte des histoires, expérimente des rencontres avec d'autres sujets, fait l'objet de désirs et d'incitations. Par conséquent, la constitution des hommes et des femmes en tant que sujets de connaissance dépend de la multiplicité des relations qu'ils développent avec le monde.

Cependant, elles circulent dans les relations collectives de travail où elles sont validées et, selon leur nature et les circonstances locales, peuvent faire l'objet de transmission et de partage avec leurs pairs. C'est la fonction et la singularité de l'enquête dialogique que de porter au langage ces manières de dire, ces manières de voir et ces manières de faire. En effet, sans espace de socialisation des récits de pratiques qui sont, par extension, des récits de vie, l'expérience singulière reste individuelle. Ainsi, par la circulation des récits du fait de la mise en dialogue de l'expérience, s'opèrent trois mouvements : un travail d'extériorisation par lequel, selon les termes de Dilthey, l'expérience en étant exprimée à la communauté s'accomplit (mouvement vital); la reconnaissance de la singularité des manières de voir et des manières de vivre (mouvement de socialisation) ; la thématisation des dimensions restées imperçues, saisies en tant qu'objets au sein des collectifs.

Pour reprendre les mots de Charlot (1999, p. 73): «Il n'y a pas de rapport avec la connaissance, mais avec celle d'un sujet. Il n'y a de sujet que dans un monde et dans une relation 
avec l'autre. Le rapport au monde ne cesse pas d'être un rapport social, même s'il est celui d'un sujet. » Il convient de mentionner que la dimension sociale de la relation avec la connaissance confère aux autres dimensions une expression singulière. L'identité, l'être social et la préférence pour l'une ou l'autre forme d'apprentissage sont entrelacés. Cependant, il faut éviter les visions déterministes entre la structure sociale et le rapport au savoir, car considérer que « [...] le rapport au savoir est social ne signifie pas qu'il doive être mis en correspondance avec une simple position sociale. La société n'est pas seulement un ensemble de positions, c'est aussi l'histoire » (CHARLOT, 1999, p. 74).

\section{Conclusion}

L'objet de cet article, corédigé par deux auteurs dans une dynamique alternant les temps d'écriture, les moments d'échanges, de dialogue et de controverse, est de formaliser les dimensions anthropologiques des modes d'enquête narratifs et dialogiques sur les savoirs au travail. Ainsi, si différentes formes d'enquête sur le travail sont possibles (THIEVENAZ, 2019), la singularité de l'approche narrative peut être maintenant résumée pour la caractériser. Tout d'abord, réfléchir aux changements dans le travail à travers les échanges entre concepts et expériences: analyser le travail en tant qu'expérience et/ou du point de vue de l'activité constitue un défi épistémologique, politique et éthique permanent. Reconnaître que les travailleurs ont de l'expérience dans les méandres du processus productif, dans les situations de travail dans lesquelles ils se trouvent, pose le problème théorique et méthodologique de l'approche et de la mise en évidence de ces expériences, de l'analyse de l'activité. Ensuite, considérer comme partenaires de ce projet de connaissance les protagonistes dans leur diversité: l'accent est mis sur la construction d'un nouveau régime de production des connaissances, à partir de la relation entre la théorie et la pratique, selon les mots de Schwartz, et de la double anticipation entre le concept et l'expérience, structurant en permanence les configurations historiques. Par ailleurs, il y a un appel à faire de la recherche sur les dispositifs de construction à trois pôles en vue de nouveaux régimes de production de la connaissance.

Ainsi, que la connaissance soit pensée à partir d'un ancrage ferme et résolu « en première personne » (ZAHAVI, 2015), comme cela est le cas pour le courant des histoires de vie en formation (PINEAU, 1984) ou dans les courants de la micro-phénoménologie (PETITMENGIN; BITBOL; OLLAGNIER-BELDAME, 2015), ou selon une perspective qui procède par le dialogisme des dispositifs de conversation avec les travailleurs - cercles de culture (FREIRE, 1996b), communauté scientifique élargie (ODDONE; BRIANTE, 1981), et dispositive dynamique à trois pôles (SCHWARTZ, 2000a, 2000b) -, l'enjeu de l'enquête vise la formation de soi des chercheurs et/ ou travailleurs, la transformation des conditions sociales par le renforcement d'identités et du pouvoir d'agir des collectifs de travail, la structuration de moyens d'intervention et d'enquête pour interroger éthiquement le vécu au travail en recherche.

\section{RÉFÉRENCES}

ADAO, L. C. et al. Conexões de saberes sobre trabalho no setor mineral. In: SEMINÁRIO NACIONAL DE EDUCAÇÃO PROFISSIONAL E TECNOLOGICA, 1., 2008, Belo Horizonte. Anais [...] Belo Horizonte: CEFET, 2008.

BILLETER, Jean-François. Leçons sur TchouangTseu. Paris: Allia, 2002.

BOUTET, Josiane. Paroles au travail. Paris: L'Harmattan, 2005.

BRETON, Hervé. Enquête sur les effets vécus au cours de l'activité biographique : vers une perspective micro-phénoménologique pour penser l'herméneutique du soi. Revista Brasileira de Pesquisa (Auto)Biográfica, Salvador, v. 3, n. 7, p. 274-289, jan./abr. 2018.

BRETON, Hervé. L'enquête narrative, entré durée et détails. Éducation Permanente, n. 222, [2020?]. 
À paraître.

BRUNER, Jérôme. Culture et modes de pensée. L'esprit humain dans ses œuvres. Paris: Retz, 1986.

DE CERTEAU, Michel. L'invention du quotidien. Tome 1: Arts de faire. Paris: Folio, 1990.

CHALMERS, David. Facing up to the problem of consciousness. Journal of Consciousness Studies, n. 2/3, p. 91-118, 1995.

CHARLOT, Bernard. Du Rapport aux Savoirs. Paris: Anthropos, 1999.

CUNHA, Daisy. Trabalho: Minas de saberes e de valores. Belo Horizonte: UFMG, 2007.

DELORY-MOMBERGER, Christine. La condition biographique. Essai sur le récit de soi dans la modernité avancée. Paris: Téraèdre, 2010.

DENOYEL, Noël. Alternance tripolaire et raison expérientielle à la lumière de la sémiotique de Peirce. Revue française de pédagogie, n. 128, p. 35-42, 1999.

FREIRE, Paulo. Pedagogia do oprimido. Rio de Janeiro: Paz e Terra, 1996a.

FREIRE, Paulo. Pedagogia da autonomia: saberes necessários à prática educativa. 30. ed. São Paulo: Paz e Terra, 1996b.

FRUTEAU DE LACLOS, Frédéric. Pour une epistemology française. Souriau et la connaissance du sens commun. Revue de Métaphysique et de Morale, n. 90, p. 177-191, 2016.

GEORGAKOPOULOU, Alexandra. Small stories, interaction and identities. Amsterdam: John Benjamins Publishing Company, 2007.

GONZÁLEZ MONTEAGUDO, José. Des approches européennes non francophones en histoires de vie. Pratiques de Formation/Analyses, n. 55, p. 9-85, 2008.

GUÉRIN, François. et al. Comprendre le travail pour transformer, la pratique de l'ergonomie. Montrouge: ANACT, 2007.

LAPLANTINE, François. La description ethnographique. Paris: Éditions Nathan, 1996.

MERLEAU-PONTY, Maurice. Le visible et l'invisible. Paris: Gallimard, 1993.

MONTANDON, Alain. Formes brèves et microrécits. Les Cahiers de Framespa, n. 14, 2013.
Disponible sur: http://journals.openedition.org/ framespa/2481. Consulté le: 4 sep. 2019.

ODDONE, Ivar; RE, Alessandra; BRIANTE, Gianni. Redécouvrir l'expérience au travail. Paris: Les Éditions sociales, 1981.

PASTRÉ, Pierre. La conceptualisation dans l'action. Bilan et nouvelles perspectives. Éducation permanente, n. 133, p. 13-35, 1999.

PETITMENGIN, Claire; BITBOL, Michel; OLLAGNIER-BELDAME, Magali. Vers une science de l'expérience vécue. Intellectica, n. 64, p. 53-76, 2015.

PINEAU, Marie-Michèle Gaston. Produire sa vie: autoformation et autobiographie. Québec: Edilig, 1984.

PINEAU, Gaston; LE GRAND, Jean-Louis. Les histoires de vie. Paris: Presses Universitaires de France, 2019.

RICCEUR, Pierre. Temps et récit. 1. L'intrigue et le récit historique. Paris: Seuil, 1983.

SCHWARTZ, Yves. Philosophie et Ergologie. Bulletin de la Société Française de Philosophie, Paris, n. 2, avr/jui, 2000a.

SCHWARTZ, Yves. Le paradigme ergologique ou un métier de philosophe. Toulouse: Octarès, 2000b.

SOUZA, Elizeu Clementino de. (Auto)biographie. Écrits de soi et formation au Brésil. Paris: L'Harmattan, 2008.

THIEVENAZ, Joris. Enquêter et apprendre au travail: approcher l'expérience avec John Dewey. Paris: Éditions et Passions, 2019.

VARELA, Francisco. Le cercle créateur. Écrits (1976-2001). Paris: Seuil, 2017.

VARELA, Francisco; THOMPSON, Eva; ROSCH, Eleonor. L'inscription corporelle de l'esprit. Paris: Seuil, 2003.

VERMERSCH, Pierre. Psycho-phénoménologie de la réduction. Alter, n. 11, p. 229-255, 2003.

ZAHAVI, Dan. Intentionnalité et phénoménalité. Un regard phénoménologique sur le problème difficile. Philosophie, n. 124, p. 80-104, 2015.

Recebido em: 01/08/2019 Aprovado em: 08/11/2019 